\title{
Reaping the processing potential of FPGA on double-precision floating-point operations: an eigenvalue solver case study
}

\author{
Miaoqing Huang \\ Computer Science \& Computer Engineering Department \\ University of Arkansas \\ Fayetteville, AR 72701, USA \\ mqhuang@uark.edu
}

\author{
Ozlem Kilic \\ Electrical Engineering \& Computer Science Department \\ The Catholic University of America \\ Washington, DC 20064, USA \\ kilic@cua.edu
}

\begin{abstract}
Many scientific applications such as electromagnatics require their operations carried out in double-precision floating-point format. The efficiency of these applications is mainly subject to the floating-point processing performance on the target processors. In this work, we use an eigenvalue solver application as a case study to demonstrate the processing potential of an FPGA device when dealing with floatingpoint operations. Relying on deep pipelines and large local memory directly accessible within the FPGA device, more than $20 \times$ performance improvement has been achieved for this particular case whose computation is intrinsically sequential. The methodology described in this paper can be conveniently applied to other domain applications that share similar data processing characteristics, demonstrating an impact on a broad range of scientific fields, e.g., numerical linear algebra.
\end{abstract}

Keywords-Double precision floating-point operations, Eigenvalue solver, Field-Programmable Gate Arrays (FPGAs), Electromagnetics

\section{INTRODUCTION}

Field-Programmable Gate Arrays (FPGAs) have been used as co-processor accelerators in many applications, e.g., cryptography, image processing and bioinformatics, showing orders of magnitude performance speedup compared with traditional microprocessors [1]. Relying on two techniques, parallelism and pipelining, hardware implementations on FPGA devices have demonstrated many advantages when dealing with integer operations and bit manipulations.

On the other hand, the floating-point performance of FPGA devices is comparatively poor, mainly due to the lack of built-in floating-point units inside the devices. Previous work has tried to address this disadvantage [2]-[8]. Several floating-point libraries consisting of basic operators, such as addition/subtraction, multiplication and division, have been reported [4], [5]. In order to reduce the high cost for implementing floating-point operators on FPGA devices, lowprecision or mixed precision approaches have been proposed and analyzed [2], [6], [8]. Further, FPGA vendors have stepped in to provide more convenient solutions. Xilinx, a large FPGA vendor, has included pipelined floating-point operators in its ISE tools [9]. The Xilinx floating-point core complies with much of the IEEE-754 Standard [10].
It supports a greater range of fraction and exponent word length than the IEEE-754 Standard, including the typical formats (e.g., single-precision and double-precision) and customized formats. In the meantime, it provides the tradeoff between the resource requirement and the performance so that the cores can fit different scenarios.

The direct support from the device and tool vendors brings the ease of use to most end-users of FPGA co-processors, i.e., domain scientists. In this work, we use an eigenvalue solver as a case study to show how remarkable performance improvement can be achieved using the basic floating-point operators generated by vendor tools.

Finding the eigenvalues of large matrices (rank $>400$ ) is a critical and computation-demanding step in many domain applications such as electromagnetics [11], [12] and image processing. In order to achieve high accuracy, numbers are generally presented in double-precision floating-point format. Typically the entry of a matrix is a complex number consisting of a real part and an imaginary part. Due to the high rank of these target matrices, numerical approaches [13] have to be adopted to solve this issue. Numerical approaches generally apply the same type of operations repeatedly on the data, which brings a potential to achieve significant performance improvement through hardware implementation.

We present two techniques in our implementation: (i) Building deep-pipelined complex processing units using basic floating-point operators. Especially a fully pipelined floating-point accumulator is described in detail. (ii) Distributing data across multiple local memory banks and carefully designing the access pattern to maximize the parallelism. The hardware implementation of our eigenvalue solver on an SGI RC100 reconfigurable computer [14] is capable of achieving significant performance improvement compared with the corresponding software implementation on an Intel Itanium 2 processor.

The remaining text is organized as follows. Section II introduces the QR eigenvalue algorithm. The hardware platform and implementation details are discussed in Section III, followed by results in Section IV. Finally, Section V concludes this work. 


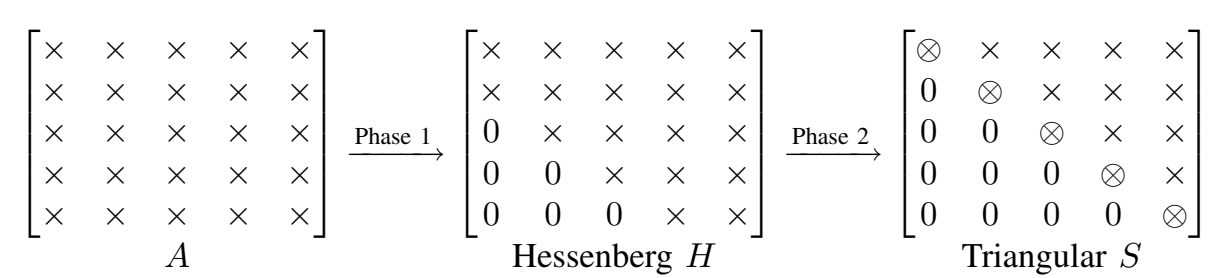

\section{QR Eigenvalue Algorithm}

Given a square matrix $A \in \mathbb{C}^{n \times n}$, an eigenvalue $\lambda$ and its associated eigenvector $\mathbf{v}$, by definition, are a pair obeying the relation $A \mathbf{v}=\lambda \mathbf{v}$. Equivalently, $(A-\lambda I) \mathbf{v}=0$ (where $I$ is the identity matrix), implying $\operatorname{det}(A-\lambda I)=0$. This determinant can be expanded into a polynomial in $\lambda$, known as the characteristic polynomial of $A$. One common method for determining the eigenvalues of a small matrix is by finding the roots of its characteristic polynomial. However, a general polynomial of order $n>4$ cannot be solved by a finite sequence of arithmetic operations and radicals. Therefore, many numerical iterative algorithms have been proposed [13] to solve the eigenvalue problem of high-rank square matrices, such as Power Method, Inverse Iteration, Jacobi Method, etc. Among these, the shifted Hessenberg QR algorithm [15]-[17] is accepted as a practical solution adopted in most applications to deal with general square matrices.

There are two phases in the practical QR algorithm, as described in (1). In the first phase, the original matrix $A$ is reduced to the upper Hessenberg form $H$ using the Householder transformation [18]. The second phase involves applying the implicit QR iteration with shifts on the unreduced Hessenberg matrix $H$ until it converges to a triangular matrix, i.e., the Schur form $S$. The eigenvalues of a triangular matrix are listed on the diagonal, i.e., the $\otimes \mathrm{s}$ in (1), and the eigenvalue problem is solved once this form is achieved.

In the most generalized case, each entry in the square matrix is a complex number consisting a real part and an imaginary part. Both real and imaginary parts are presented in double-precision floating-point format. This assumption is used in the remaining part of this work. The other assumption is the starting value of the subscripts, which is used to specify the position of each entry in the matrix. We assume that the subscript starts from zero. In other words, for an $n \times n$ matrix $A$, the top left entry and the bottom right entry are denoted as $A_{0,0}$ and $A_{n-1, n-1}$, respectively.

\section{IMPLEMENTATION ON THE SGI RC100 RECONFIGURABLE COMPUTER}

The QR eigenvalue algorithm is implemented on the SGI RC100 reconfigurable computer as a hardware/software codesign. We first briefly introduce the platform, then discuss the hardware design part in detail.
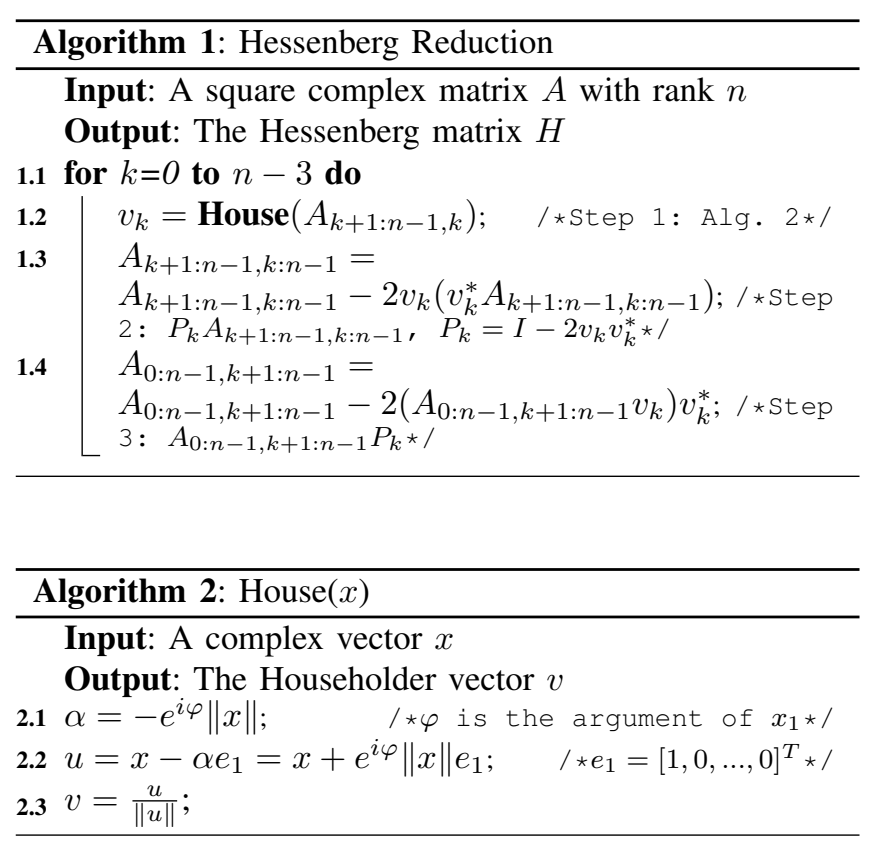

\section{A. Platform}

SGI's Altix RASC RC100 reconfigurable computer is a blade-based heterogeneous supercomputer in which NUMAlink ${ }^{\mathrm{TM}} 4$ interconnect is used to connect different types of computing blades, as shown in Fig. 1(a). Each blade itself is a homogeneous node consisting of the same type of processors, i.e., either the microprocessor or the FPGA co-processor. The Altix 450 at The Catholic University of America comprises two microprocessor blades and one FPGA blade. The detailed architecture of a RASC RC100 FPGA blade is shown in Fig. 1(b). There are two FPGA devices on one single RASC blade. Each FPGA device, Xilinx Virtex-4LX200, is equipped with 5 banks of SRAM for local data storage. The size of each SRAM bank is 8 MB. Every bank has separate 64-bit reading and writing ports directly connected to the FPGA device. In other words, the FPGA device can access these 5 local memory banks concurrently; further, it can read from and write to the same memory bank simultaneously. Besides the local memory, each FPGA device is capable of communicating with microprocessor blades through NUMAlink ${ }^{\mathrm{TM}} 4$ interconnect, achieving a theoretical $3.2 \mathrm{~GB} / \mathrm{s}$ on both directions. 


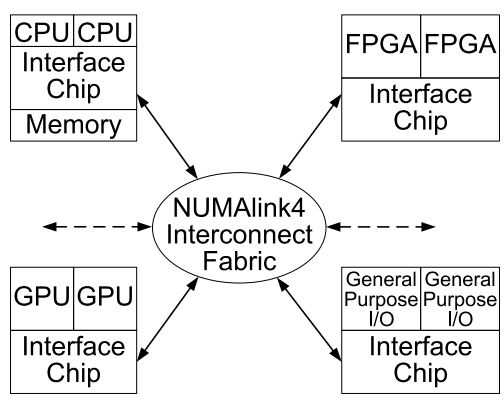

(a) Multi-paradigm computing

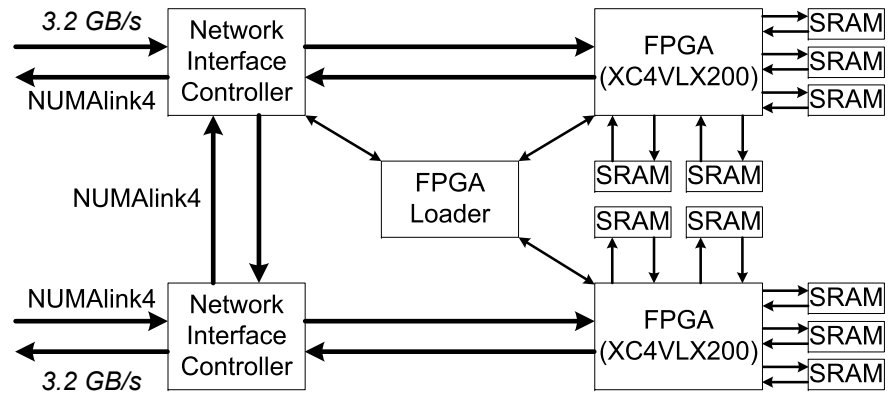

(b) SGI RASC RC100 FPGA blade architecture

Figure 1. SGI Altix RASC RC100 reconfigurable computer

\section{B. Hardware Implementation of the QR Algorithm}

In the hardware implementation of the $\mathrm{QR}$ eigenvalue algorithm on the FPGA device, we combine two physical local memory banks into a 128-bit wide logical memory bank so that each memory entry can store one complete matrix entry. Therefore, the real part and the imaginary part of a complex value can be accessed simultaneously.

As described in Section II, there are two phases in the QR algorithm. These two phases are implemented in two separate FPGA configurations. The first phase, Hessenberg reduction, is carried out by applying the Householder reflection for $n-2$ iterations (see Alg. 1), where $n$ is the rank of the original matrix $A$. Each iteration comprises three steps, as shown in Table I. Each step further includes multiple sub-steps. In our hardware design, Steps 1, 2 and 3 comprise 4, 3 and 3 sub-steps, respectively. The steps in each iteration, and the sub-steps within every step are carried out sequentially due to data dependencies. The advantage of hardware implementation comes from the parallel processing within each sub-step. For example, Sub-step 1.1 involves multiplication, addition, accumulation and square root operations to calculate the norm of a vector. If all the basic operators, e.g., multipliers and adders, are fully pipelined, it will take roughly $n-k-1$ clock cycles to finish this sub-step (if we ignore all potential latencies). By putting everything together, the total number of clock cycles required to reduce a matrix of rank $n$ to its Hessenberg form can thus be computed as:

$$
\sum_{k=0}^{n-3}\left(3 k^{2}-9 n k+6 n^{2}-3 n-2\right)=\frac{5}{2} n^{3}-\frac{9}{2} n-11 .
$$

The second phase of the QR algorithm is to convert the upper Hessenberg matrix to its upper triangular form, which is implemented as a hardware/software co-design (see pp. 359 in [19] for detailed description). The main step of the second phase is the Francis QR Step, in which the most computationing demanding part is implemented in hardware

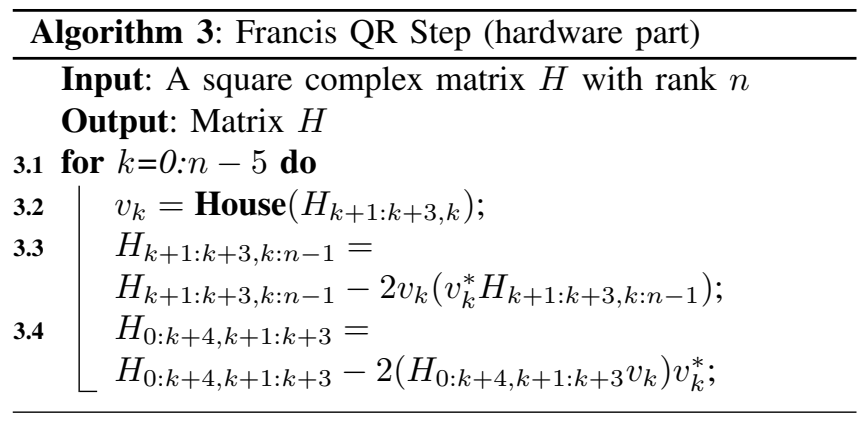

as a separate FPGA configuration. Its functionality is shown in Alg. 3. By comparing Alg. 1 and Alg. 3, it can be found that Alg. 3 is a shrunk version of Alg. 1. In other words, the implementation of both algorithms will share the majority of their logic such as the floating-point operators and the control flow. Some small modifications are required to reduce the scope of the computation in Alg. 1 to match the functionality of Alg. 3. In general, the computation in the Francis QR Step is significantly less than the computation in the Hessenberg reduction phase. By applying the same time analysis on the Francis QR Step, the time (in terms of clock cycle) for each step is shown in Table I. The total number of clock cycles required to perform the Francis QR Step can be computed as:

$$
\sum_{k=0}^{n-5}(9 n+55)=9 n^{2}+19 n-220 .
$$

1) Floating-point Operator Design: These doubleprecision floating-point operators, i.e., multiplier, adder, subtractor, divider and square rooter, were generated by using CORE Generator [9] from the Xilinx ISE package. These operators are all fully pipelined so that they are capable of accepting new operands every clock cycle. The generic diagram of a floating-point operator is shown in Fig. 2. Besides the operands and result signals, two extra signals, 
Table I

CALCULATION BREAKDOWN OF ITERATION $k$ IN THE QR ALGORITHM

\begin{tabular}{|c|c|c|c|c|}
\hline Step & Sub-step & Calculation & Number of clo & cycles for computation $^{\dagger}$ \\
\hline \multicolumn{5}{|c|}{ Hessenberg Reduction } \\
\hline \multirow{4}{*}{1} & 1.1 & $\left|x\|,\| x_{1}\right|$ & $n-k-1$ & \multirow{10}{*}{$3 k^{2}-9 n k+6 n^{2}-3 n-2$} \\
\hline & 1.2 & $x_{1 r}+\|x\| \cos \varphi, x_{1 i}+\|x\| \sin \varphi$ & 1 & \\
\hline & 1.3 & $\|u\|$ & $n-k-1$ & \\
\hline & 1.4 & $u /\|u\|$ & $n-k-1$ & \\
\hline \multirow{3}{*}{2} & 2.1 & $m=v_{k}^{*} A_{k+1: n-1, k: n-1}$ & $(n-k)(n-k-1)$ & \\
\hline & 2.2 & $N=v_{k} m$ & $(n-k)(n-k-1)$ & \\
\hline & 2.3 & $A_{k+1: n-1, k: n-1}-2 N$ & $(n-k)(n-k-1)$ & \\
\hline \multirow{3}{*}{3} & 3.1 & $m^{\prime}=A_{0: n-1, k+1: n-1} v_{k}$ & $n(n-k-1)$ & \\
\hline & 3.2 & $N^{\prime}=m^{\prime} v_{k}^{*}$ & $n(n-k-1)$ & \\
\hline & 3.3 & $A_{0: n-1, k+1: n-1}-2 N^{\prime}$ & $n(n-k-1)$ & \\
\hline \multicolumn{5}{|c|}{ Francis QR Step } \\
\hline \multirow{4}{*}{1} & 1.1 & $\left|x\|,\| x_{1}\right|$ & 3 & \multirow{10}{*}{$9 n+55$} \\
\hline & 1.2 & $x_{1 r}+\|x\| \cos \varphi, x_{1 i}+\|x\| \sin \varphi$ & 1 & \\
\hline & 1.3 & $\|u\|$ & 3 & \\
\hline & 1.4 & $u /\|u\|$ & 3 & \\
\hline \multirow{3}{*}{2} & 2.1 & $m=v_{k}^{*} H_{k+1: k+3, k: n-1}$ & $3(n-k)$ & \\
\hline & 2.2 & $N=v_{k} m$ & $3(n-k)$ & \\
\hline & 2.3 & $H_{k+1: k+3, k: n-1}-2 N$ & $3(n-k)$ & \\
\hline \multirow{3}{*}{3} & 3.1 & $m^{\prime}=H_{0: k+4, k+1: k+3} v_{k}$ & $3(k+5)$ & \\
\hline & 3.2 & $N^{\prime}=m^{\prime} v_{k}^{*}$ & $3(k+5)$ & \\
\hline & 3.3 & $H_{0: k+4, k+1: k+3}-2 N^{\prime}$ & $3(k+5)$ & \\
\hline
\end{tabular}

$\dagger$ Ignoring all latencies.

Table II

RESOURCE REQUIREMENT AND LATENCY OF FULLY PIPELINED FLOATING-POINT OPERATORS

\begin{tabular}{l|c|c|c}
\hline & Slices & XtremeDSP48 & Latency \\
\hline \hline Multiplier & 1,332 & 9 & 17 \\
\hline Adder/Subtractor & 1,274 & 0 & 14 \\
\hline Divider & 3,335 & 0 & 57 \\
\hline Square rooter & 1,904 & 0 & 57 \\
\hline
\end{tabular}

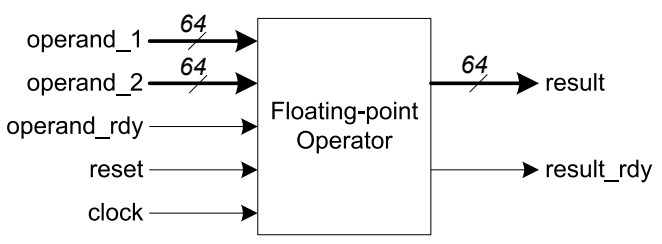

Figure 2. Generic diagram of a floating-point operator

operand_rdy and result_rdy, are used to indicate the validity of the input and the output respectively. By having these two signals, it is convenient to chain multiple operators to form a deep pipeline. The resource requirements of these operators are listed in Table II. Among them, only the multiplier requires the use of the built-in XtremeDSP48, for which the target FPGA device Xilinx Virtex-4LX200 has 96 in total.

One necessary operator in the matrix computation is a fully pipelined accumulator used in Step 1.1, 1.3, 2.1 and 3.1 in Table I. These accumulators are used to either calculate the norm of a vector or perform the multiplication between a row (of the first matrix) and a column (of the second matrix).
Construction of full-pipelined floating-point accumulators has been a research topic for many years [20], [21]. In this work, we propose a simple approach to build a fully pipelined accumulator on top of multiple adders. The internal architecture of the fully pipelined accumulator is shown in Fig. 3. It consists of $\left\lceil\log _{2} L\right\rceil+2$ fully pipelined adders, where $L$ is the latency of the adder. In this architecture, the adder next to the last one carries out the accumulation. We use symbol $A \_a c c$ to specify this adder. Because it has the latency of $L$ clock cycles, it is not able to take any new input once the addition between the intermediate sum and the previous input is in process. However, it is required that the composed accumulator is capable of accepting new operand every clock cycle. To make sure that the interval between two inputs to the $A_{-} a c c$ is equal to or larger than the processing latency $L$ and no operand gets dropped, an array of $\left\lceil\log _{2} L\right\rceil$ adders are concatenated in front of the $A \_a c c$. Because each adder will reduce two inputs to one output, the data arrival rate is dropped to half after one addition, as the pattern shown in Fig. 4. Therefore, if a chain of $\left\lceil\log _{2} L\right\rceil$ adders is built in front of $A_{-} a c c$, it is guaranteed that the data arrival interval to $A_{-} a c c$ is greater than or equal to its latency since $2^{\left\lceil\log _{2} L\right\rceil} \geq L$.

One extra input signal with the accumulator is op_last, which indicates the arrival of the last data item to be accumulated. This signal will travel down through the shift registers. If the over quantity of data items is odd, the last item will be added with zero, which is realized using the multiplexer. Since the last item or its sum with other 


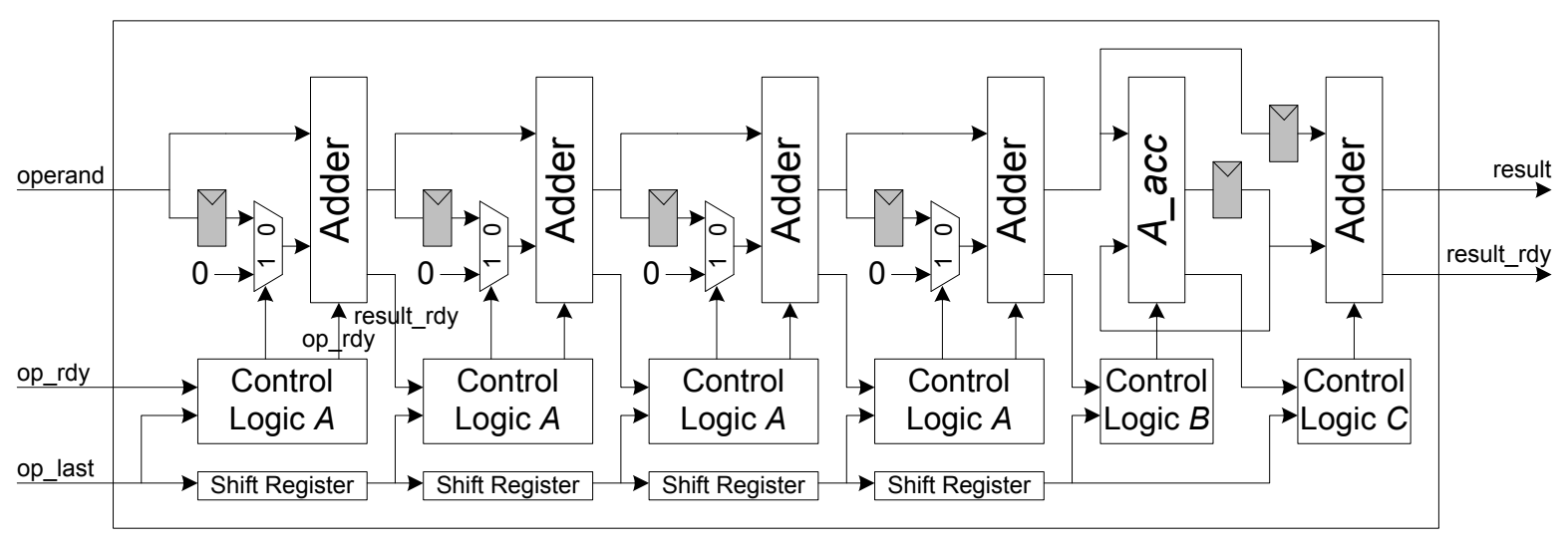

Figure 3. The internal architecture of the fully pipelined accumulator built on top of adders

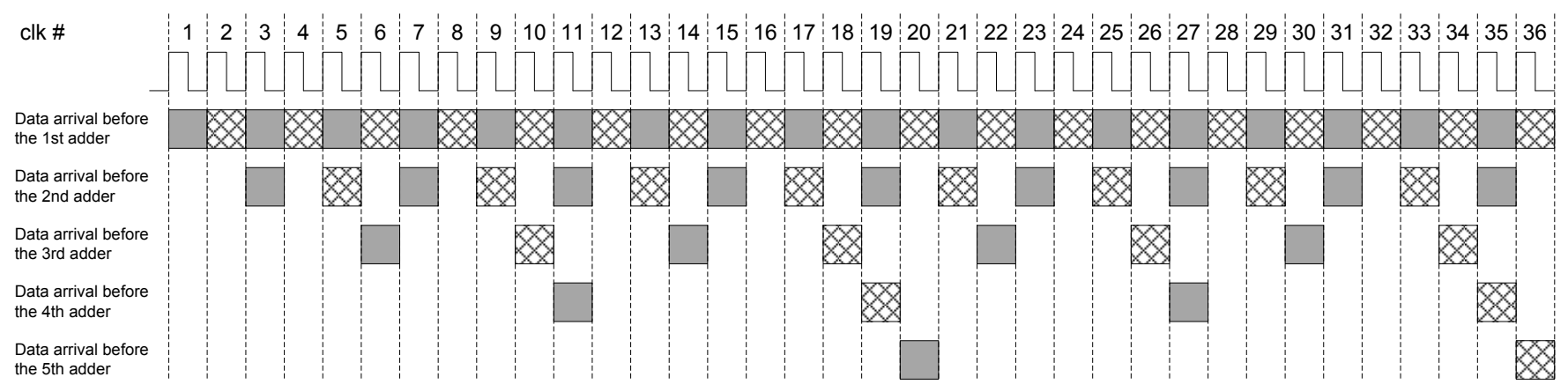

Figure 4. The data arrival pattern inside the chain of adders (assuming the latency of each adder is 1 clock cycle)
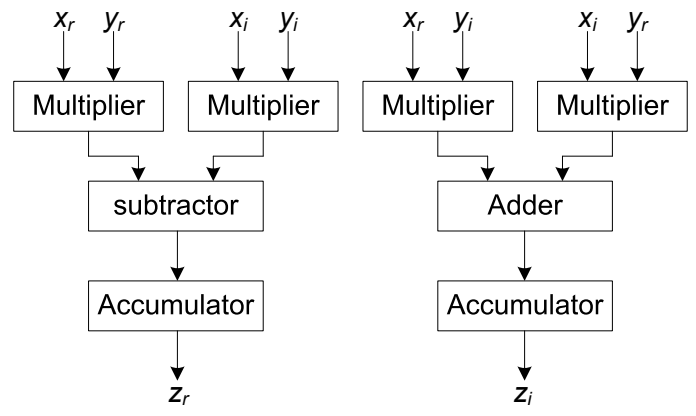

Figure 5. Use basic operators to build the complex processing unit used in matrix multiplication (with deep pipeline)

data items is pushed down to the pipeline without delay, it may arrive at the $A \_a c c$ when $A \_a c c$ is in the middle of accumulation. In this case, it is temporarily saved in the register as one input of the last adder. Once the intermediate accumulating result becomes available, it is added to the last item to produce the final result. One shortcoming of this design is that the interval between two op_last signals has to be greater than the latency $L$. Otherwise, the data between two consecutive accumulations may mix together.

Having either generated these basic operators or built the complex units such as accumulator, we can use them to compose deep pipelines to handle more complicated operations. Fig. 5 demonstrates the composition of the processing unit used in matrix multiplication, i.e., $C=A \times B$. Each entry in $C$ is the product of a row in $A$ and a column in $B$, i.e., $C_{i, j}=\sum_{k=0}^{n-1} A_{i, k} B_{k, j}$. Because every entry is a complex number, four multipliers, one subtractor, one adder, and two accumulators are required to build this unit.

2) Local Memory Utilization: Local memory plays an equally important role in the hardware design for the eigenvalue solver as the available number of memory banks and their access ports will affect the performance significantly.

As mentioned at the beginning of Section III-B, two physical memory banks are combined to form a logical local memory bank so that the size of a memory entry matches the size of a matrix entry. When storing a matrix in the memory, we store the matrix row by row from top to bottom. Within each row, those entries are stored from left to right in a sequence.

In our implementation, two 128-bit wide local memory banks are used, each of which has a size of $16 \mathrm{MB}$. Each memory bank is divided evenly into 4 regions*. In order to maximize the data processing parallelism, we distributed

* Only the first two regions, region 0 and region 1 , are used in the design. 


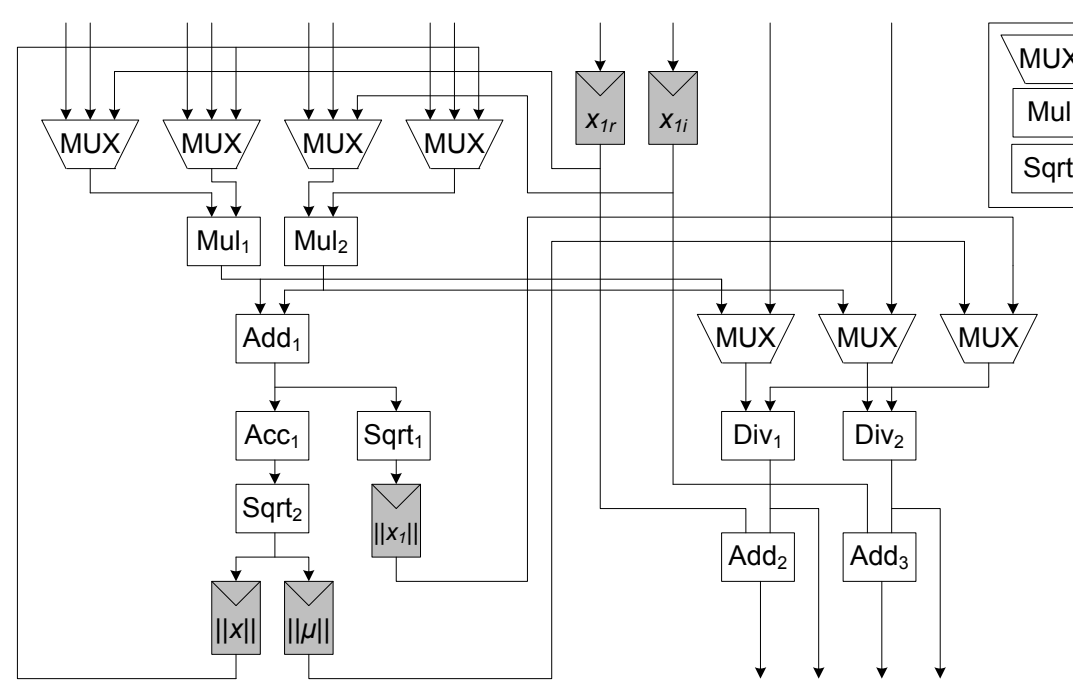

(a)

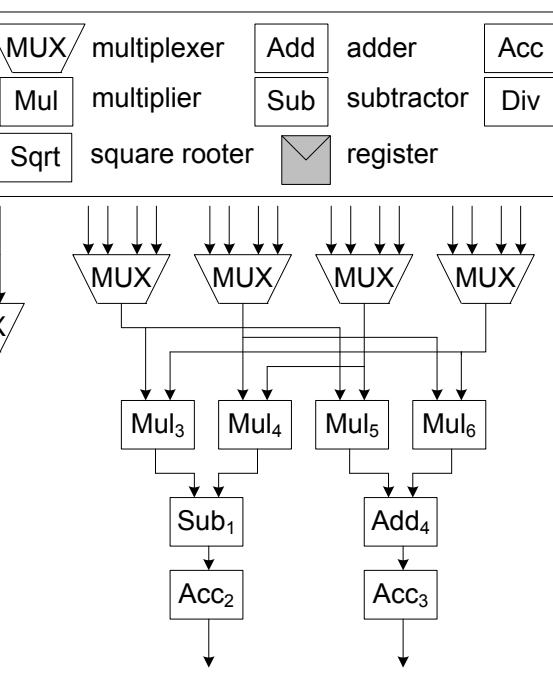

(b)

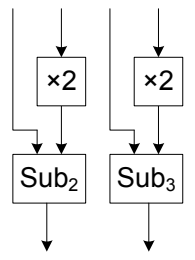

(c)

Figure 6. The computing blocks in the hardware implementaion: (a) the computing block used in Step 1; (b) the computing block used in Step 2.1, 2.2, $3.1,3.2 ;$ (c) the computing block used in Step 2.3, 3.3. (Note: (1) all inputs and outputs are connected to the local memory interface; (2) the control logic is not illustrated in the figure)

Table III

LOCAL MEMORY ACCESS DIRECTION AND DATA DISTRIBUTION

\begin{tabular}{c|c|c|c|c}
\hline \multirow{2}{*}{ Sub-step p } & \multicolumn{2}{|c|}{ bank 0 } & \multicolumn{2}{c}{ bank 1 } \\
\cline { 2 - 5 } & read & write & read & write \\
\hline 1.1 & region $0(A)$ & $\times$ & $\times$ & region $0(x)$ \\
\hline 1.2 & $\times$ & $\times$ & $\times$ & region $0(\mu)$ \\
\hline 1.3 & $\times$ & $\times$ & region 0 $(\mu)$ & $\times$ \\
\hline 1.4 & $\times$ & $\times$ & region 0 $(\mu)$ & region 0 $\left(v_{k}\right)$ \\
\hline 2.1 & region $0(A)$ & region $1(m)$ & region 0 $\left(v_{k}\right)$ & $\times$ \\
\hline 2.2 & region 1 $(m)$ & $\times$ & region 0 $\left(v_{k}\right)$ & region $1(N)$ \\
\hline 2.3 & region 0 $(A)$ & region $0(A)$ & region 1 $(N)$ & $\times$ \\
\hline 3.1 & region $0(A)$ & region $1\left(m^{\prime}\right)$ & region 0 $\left(v_{k}\right)$ & $\times$ \\
\hline 3.2 & region $1\left(m^{\prime}\right)$ & $\times$ & region 0 $\left(v_{k}\right)$ & region $1\left(N^{\prime}\right)$ \\
\hline 3.3 & region $0(A)$ & region $0(A)$ & region 1 $\left(N^{\prime}\right)$ & $\times$ \\
\hline
\end{tabular}

the data (including the original data and the intermediate data) accordingly between these two local memory banks so that there is no competition regarding the memory access port within each sub-step. In the hardware implementation, the two most significant bits of the address signal are used to select the region inside a memory bank to access. Their value are decided based on Table III and go through the same pattern each iteration.

From Table III it can be observed that the data written into a region will be read out in the following sub-step in most cases. In order to overcome this read after write (RAW) hazard [22], 16 clock cycles are awaited after the writing command for the last data block is issued. Then the computation moves to the next sub-step and start reading from the same region.

3) The Overall Design: The detailed hardware implementation of the computing blocks is illustrated in Fig. 6. Since multiple steps have to be carried out sequentially, many basic computing units are re-used to reduce the resource cost. For example, the pipeline chain consisting of $M u l_{1}, M u l_{2}$, $A d d_{1}, A c c_{1}$ and $S q r t_{2}$ are re-used in Step 1.1 and Step 1.3 to compute $\|x\|$ and $\|\mu\|$, respectively. $\cos \varphi$ and $\sin \varphi$ are calculated on the fly by using division, i.e., $x_{1 r} /\left\|x_{1}\right\|$ and $x_{1 i} /\left\|x_{1}\right\|$. Therefore, the outputs of Step 1.2 correspond to the output of $A d d_{2}$ (i.e., $x_{1 r}+\|x\| \cdot x_{1 r} /\left\|x_{1}\right\|$ ) and $A d d_{3}$ (i.e., $x_{1 i}+\|x\| \cdot x_{1 i} /\left\|x_{1}\right\|$ ). The multiplication between matrix/vector and vector/vector in Step 2.1, 2.2, 3.1 and 3.2 is realized using the pipeline chain in Fig. 6(b). Both the real part and the imaginary part of a complex entry are computed simultaneously. The control logic is not illustrated in Fig. 6. It is mainly composed by three components, i.e., (1) a finite state machine whose statuses represent different steps and sub-steps, (2) the logic to generate correct reading and writing address for memory access, and (3) the logic to control the operations of the units in Fig. 6.

\section{REsults}

The hardware implementation of the Hessenberg reduction occupies 56,520 (63\%) slices on the target FPGA device and runs at $100 \mathrm{MHz}$. The rank of the object matrix is passed to the hardware design as a parameter through a register. Before the FPGA starts processing, the original matrix as well as its rank are transferred from the host to the FPGA. After the processing is finished, the upper Hessenberg matrix is transferred back to the host memory. We tested matrices of different ranks and collected their corresponding hardware computation times, as listed in Table IV. The hardware 
Table IV

PERFORMANCE IMPROVEMENT OF THE HESSENBERG REDUCTION

\begin{tabular}{|c|c|c|c|c|c|c|c|c|c|c|c|}
\hline \multirow{2}{*}{$\begin{array}{c}\text { Matrix } \\
\text { Rank }\end{array}$} & \multicolumn{2}{|c|}{ Computation Time (s) } & \multirow{2}{*}{ Speedup } & \multirow{2}{*}{$\begin{array}{c}\text { Matrix } \\
\text { Rank } \\
\end{array}$} & \multicolumn{2}{|c|}{ Computation Time (s) } & \multirow{2}{*}{ Speedup } & \multirow{2}{*}{$\begin{array}{c}\text { Matrix } \\
\text { Rank }\end{array}$} & \multicolumn{2}{|c|}{ Computation Time (s) } & \multirow{2}{*}{ Speedup } \\
\hline & Hardware* & Software & & & Hardware* & Software & & & Hardware $^{*}$ & Software & \\
\hline 20 & 0.007 & 0.004 & 0.64 & 180 & 0.161 & 3.078 & 19.11 & 340 & 1.019 & 20.764 & 20.38 \\
\hline 40 & 0.008 & 0.034 & 4.08 & 200 & 0.217 & 4.224 & 19.45 & 360 & 1.206 & 24.648 & 20.44 \\
\hline 60 & 0.013 & 0.114 & 8.94 & 220 & 0.285 & 5.624 & 19.70 & 380 & 1.415 & 29.000 & 20.49 \\
\hline 80 & 0.021 & 0.269 & 12.83 & 240 & 0.367 & 7.300 & 19.87 & 400 & 1.647 & 33.845 & 20.55 \\
\hline 100 & 0.034 & 0.526 & 15.38 & 260 & 0.464 & 9.283 & 20.02 & 420 & 1.904 & 39.198 & 20.59 \\
\hline 120 & 0.054 & 0.910 & 16.99 & 280 & 0.576 & 11.592 & 20.13 & 440 & 2.185 & 45.106 & 20.64 \\
\hline 140 & 0.080 & 1.447 & 18.00 & 300 & 0.705 & 14.262 & 20.24 & 460 & 2.493 & 51.719 & 20.74 \\
\hline 160 & 0.116 & 2.161 & 18.66 & 320 & 0.852 & 17.309 & 20.31 & 480 & 2.829 & 59.035 & 20.86 \\
\hline
\end{tabular}

*Including data transportation time and data processing time.

computation time consists of both data transportation time ${ }^{\dagger}$ and data processing time. It is found that the measured time matches the estimation using (2) in all cases.

For comparison of acceleration over a pure software based implementation, we coded the Hessenberg reduction phase in $\mathrm{C}++$ and ran it on a workstation with Itanium 2 using a $1.66 \mathrm{GHz}$ microprocessor. The speedup between hardware and software implementations is listed in Table IV. The performance improvement is mainly due to two factors. (i) The hardware implementation is fully pipelined, which means that multiple operations can be processed concurrently. On the other hand, the microprocessor has to process these operations sequentially. (ii) FPGA devices are equipped with large amount of directly accessible local memory, e.g., 40 MB on Altix RASC RC100. The local memory of FPGA devices can be compared to the L1/L2 cache of microprocessors, which are much smaller in terms of capacity. As we can see from Alg. 1, the Hessenberg reduction operation spans on all the matrix, along both columns and rows. Since the local memory of the FPGA device is quite large, it is able to accommodate the whole matrix ${ }^{\ddagger}$. On the other hand, the Hessenberg reduction operation on the microprocessor is accompanied by frequent data swapping among the $\mathrm{L} 1$ cache (16KB), the L2 cache (256KB) [23] and the main memory, which contributes overhead.

The hardware implementation of Alg. 3 takes almost the same amount of resources (i.e., 56,327 (63\%) slices) on the FPGA device and runs at the same frequency. The interface to the second bitstream is same as the first one. We applied the same type of comparison between the hardware implementation and the software version on the Francis QR Step, as shown in Fig. 7. For a $480 \times 480$ matrix, the computation time is $0.450 \mathrm{~s}$ for software and $0.068 \mathrm{~s}$ for hardware, respectively. In other words, the hardware implementation outperforms the corresponding software version by 6.6 folds for the typical size of matrices in electromagnetics. The comparatively small performance improvement on the Fran-

\footnotetext{
${ }^{\dagger}$ It takes about $3 \mathrm{~ms}$ to transfer a $480 \times 480$ matrix back and forth between the microprocessor and the FPGA.

${ }^{\ddagger}$ The size of a $480 \times 480$ complex matrix is close to $4 \mathrm{MB}$.
}

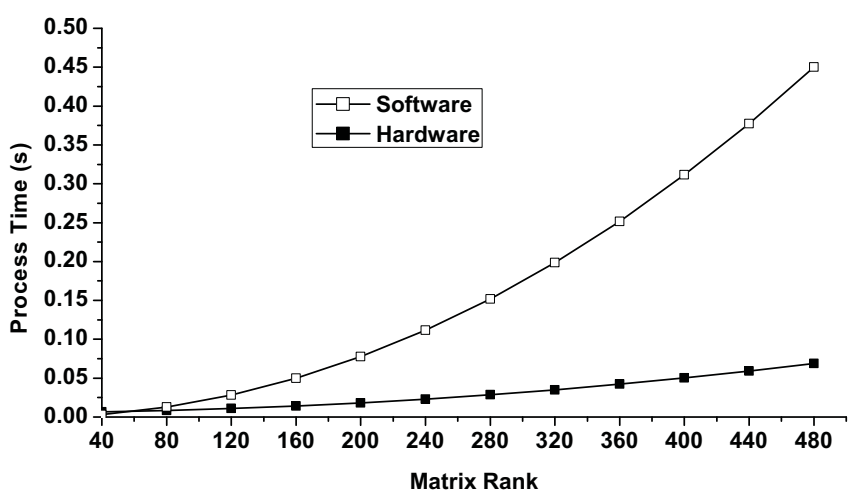

Figure 7. Performance comparison between hardware implementation and software implementation on the Francis QR step

cis QR Step is mainly due to the dramatic reduction of computation in Alg. 3. For example, the computation in line 3.4 in Alg. 3 only involves $3 k+15$ matrix entries. The corresponding line (i.e., line 1.4) in Alg. 1 involves $\mathcal{O}\left(n^{2}\right)$ matrix entries. Therefore, the advantage of a deep pipeline is more evident in the hardware implementation for the Hessenberg reduction. In the meantime, we can expect from Fig. 7 that the hardware implementation can achieve more performance improvement as the matrix rank increases because the processing time of software implementation climbs much faster than the hardware version.

\section{Conclusion}

The unsatisfying floating-point performance of the FPGA devices has long prevented them from becoming efficient co-processors in many scientific domains such as electromagnetics. In this work, we use an eigenvalue solver as a case study to demonstrate how to tackle this issue. Two techniques are used to improve the floating-point performance of the FGPA co-processor. (i) We constructed fully pipelined complex processing units by composing basic floating-point operators into deep pipeline chains. These basic operators are generated using the off-the-shelf tool, CORE Generator with Xilinx ISE. (ii) We carefully distributed the data across 
multiple local memory banks and designed the data access pattern so that those complex processing units can be efficiently used. More than 20 folds speedup has been achieved for this case and the same methodology can be easily adopted to apply on a broad range of scientific applications such as numerical linear algebra.

One of the future work we plan to carry out is to implement the memory intensive eigenvalue solver and other matrix applications on new microprocessors, which consist of L2 cache at MB level, and on other accelerators such as GPGPU. The results of these new experiments will be compared with the hardware implementation on the FPGA devices and analyzed in detail.

\section{ACKNOWLEDGMENT}

The authors would like to thank Charles Conner with The Catholic University of America for the help on the software implementation of the Hessenberg reduction, and Dr. David Andrews with University of Arkansas for valuable comments and suggestions to improve the quality of this paper.

\section{REFERENCES}

[1] T. El-Ghazawi, E. El-Araby, M. Huang, K. Gaj, V. Kindratenko, and D. Buell, "The promise of high-performance reconfigurable computing," IEEE Computer, vol. 41, no. 2, pp. 78-85, Feb. 2008.

[2] G. Lienhart, A. Kugel, and R. Männer, "Using floating-point arithmetic on FPGAs to accelerate scientific N-body simulations," in Proc. the 10th Annual IEEE Symposium on FieldProgrammable Custom Computing Machines (FCCM'02), Apr. 2002, pp. 182-191.

[3] S. Paschalakis and P. Lee, "Double precision floating-point arithmetic on FPGAs," in Proc. 2003 IEEE International Conference on Field-Programmable Technology (FPT'03), Dec. 2003, pp. 352-358.

[4] G. Govindu, R. Scrofano, and V. K. Prasanna, "A library of parameterizable floating-point cores for FPGAs and their application to scientific computing," in Proc. The International Conference on Engineering Reconfigurable Systems and Algorithms (ERSA'05), Jun. 2005, pp. 137-145.

[5] K. S. Hemmert and K. D. Underwood, "Open source high performance floating-point modules," in Proc. the 14th Annual IEEE Symposium on Field-Programmable Custom Computing Machines (FCCM'06), Apr. 2006, pp. 349-350.

[6] L. Zhuo and V. K. Prasanna, "Scalable and modular algorithms for floating-point matrix multiplication on reconfigurable computing systems," IEEE Trans. Parallel Distrib. Syst., vol. 18, no. 4, pp. 433-448, Apr. 2007.

[7] J. Detrey and F. de Dinechin, "Parameterized floating-point logarithm and exponential functions for FPGAs," Microprocessors and Microsystems, vol. 31, no. 8, pp. 537-545, Dec. 2007.

[8] J. Sun, G. D. Peterson, and O. O. Storaasli, "Highperformance mixed-precision linear solver for FPGAs," IEEE Trans. Comput., vol. 57, no. 12, pp. 1614-1623, Dec. 2008.
[9] Floating-Point Operator v3.0, Xilinx, Inc., Sep. 2006.

[10] IEEE 754-2008 Standard for Floating-Point Arithmetic.

[11] M. G. Moharam, D. A. Pommet, E. B. Grann, and T. K. Gaylord, "Stable implementation of the rigourous coupled-wave analysis for surface relief gratings: enhanced transmittance matrix approach," Journal of the Optical Society of America A, vol. 12, no. 5, pp. 1077-1086, 1995.

[12] P. Lalanne, "Improved formulation of the coupled-wave method for two-dimensional gratings," Journal of the Optical Society of America A, vol. 14, no. 7, pp. 1592-1598, 1997.

[13] J. W. Demmel, Applied Numerical Linear Algebra. Philadelphia, PA: Society for Industrical and Applied Mathematics (siam), 1997.

[14] Reconfigurable Application-Specific Computing User's Guide (007-4718-007), Silicon Graphics, Inc., Jan. 2008.

[15] J. G. F. Francis, "The QR transformation, I," The Computer Journal, vol. 4, no. 3, pp. 265-271, 1961.

[16] - "The QR transformation, II," The Computer Journal, vol. 4, no. 4, pp. 332-345, 1962.

[17] V. N. Kublanovskaya, "On some algorithms for the solution of the complete eigenvalue problem," USSR Computational Mathematics and Mathematical Physics, vol. 1, no. 3, pp. 637-657, 1963.

[18] A. S. Householder, "Unitary triangularization of a nonsymmetric matrix," Journal of the ACM, vol. 5, no. 4, pp. 339342, Oct. 1958.

[19] G. H. Golub and C. F. V. Loan, Matrix Computations (3rd edition). Baltimore, MD: The John Hopkins University Press, 1996.

[20] Z. Luo and M. Martonosi, "Accelerating pipelined integerand floating-point accumulations in configurable hardware with delayed addition techniques," IEEE Trans. Comput., vol. 49, no. 3, pp. 208-218, Mar. 2000.

[21] S. Sun and J. Zambreno, "A floating-point accumulator for FPGA-based high performance computing applications," in Proc. International Conference on Field-Programmable Technology, 2009 (FPT 2009), Dec. 2009, pp. 493-499.

[22] J. L. Hennessy and D. A. Patterson, Computer architecture: a quantitative approach, 4th Edition. Amsterdam, Boston: Morgan Kaufmann, 2006.

[23] Dual-Core Update to the Intel Itanium 2 Processor Reference Manual, Intel Corporation, Jan. 2006. 